\title{
Erratum: State-selective intersystem crossing in nitrogen-vacancy centers [Phys. Rev. B 91, 165201 (2015)]
}

\author{
M. L. Goldman, M. W. Doherty, A. Sipahigil, N. Y. Yao, S. D. Bennett, N. B. Manson, A. Kubanek, and M. D. Lukin \\ (Received 6 July 2017; published 25 July 2017)
}

DOI: 10.1103/PhysRevB.96.039905

We have become aware that the vibrational overlap function $F(\Delta)$, which is defined in Eq. (5), was incorrectly normalized. This quantity factors into the intersystem crossing (ISC) rates $\Gamma_{A_{1}}$ and $\Gamma_{E_{1,2}}$ from the states $\left|A_{1}\right\rangle$ and $\left|E_{1,2}\right\rangle$ [Eqs. (6) and (13), respectively]. As a result, the theoretical value of $\Gamma_{A_{1}}$ that is plotted in Fig. 5 is too large by a factor of $2 \pi$. We thank A. Gali for bringing this error to our attention.

We have also reexamined an assumption regarding the transverse spin-orbit coupling rate $\lambda_{\perp}$ and found it not to be valid. The dynamic Jahn-Teller effect in the ${ }^{3} E$ excited state manifold causes the strengths of the spin-orbit interaction and the excited states' response to externally applied fields to be reduced [1] by a quantity known as the Ham reduction factor [2]. This factor changes the value of $\lambda_{\perp}$ that also enters into both intersystem crossing (ISC) rates. We had previously assumed that this effect is negligible, but we discovered this not to be the case when we explicitly calculated the Ham reduction factor. The magnitude of this effect for the NV center and its implications for the ISC are topics of current theoretical interest, so we present our explicit calculation of the Ham reduction factor here.

\section{INTRODUCTION TO DYNAMIC JAHN-TELLER EFFECT}

The ${ }^{3} E$ excited state manifold exhibits the dynamic Jahn-Teller (JT) effect, which means that $E$-symmetric lattice vibration modes couple to the two degenerate, $E$-symmetric electronic orbital states $[1,3]$. We now calculate explicitly how this coupling impacts the transverse spin-orbit coupling rate $\lambda_{\perp}$, which is a crucial input to our model of the ISC mechanism.

Let $\{|X\rangle,|Y\rangle\}$ and $(x, y)$ be the orbital electronic states of the triplet ${ }^{3} E$ manifold and the generalized displacement coordinates of a set of pseudolocal $E$-symmetric phonon modes, respectively. In the absence of the JT interaction, the vibrational equations for the phonon modes corresponding to each orbital state are identical and separable, and have the form

$$
\left[-\frac{\hbar}{2 M} \nabla^{2}+\frac{1}{2} k\left(x^{2}+y^{2}\right)\right]\left|\chi_{i}(x, y)\right\rangle=\epsilon_{i}\left|\chi_{i}(x, y)\right\rangle
$$

where $M$ is the carbon atomic mass, $k$ is the harmonic spring constant of the phonon modes, and $\left|\chi_{i}\right\rangle$ is the $i$ th vibrational solution with energy $\epsilon_{i}=\left(v_{i}+1 / 2\right) \hbar(k / M)^{1 / 2}$ determined by the total number of quantum excitations $v_{i}$; this is simply the standard solution to the two-dimensional harmonic oscillator. It follows that the vibronic states of the ${ }^{3} E$ manifold have well defined electronic and vibrational quantum numbers,

$$
\Psi_{X, i}=|X\rangle \otimes\left|\chi_{i}\right\rangle, \quad \Psi_{Y, i}=|Y\rangle \otimes\left|\chi_{i}\right\rangle .
$$

In the presence of the JT interaction, however, the vibrational equations of the $|X\rangle$ and $|Y\rangle$ orbital states become linearly coupled and cannot be solved separately. Consequently, the vibronic states no longer have well-defined electronic and vibrational quantum numbers. The $i$ th vibronic state is now

$$
\left|\Psi_{i}\right\rangle=|X\rangle \otimes\left|\chi_{i, x}\right\rangle+|Y\rangle \otimes\left|\chi_{i, y}\right\rangle
$$

and satisfies the equation

$$
\left\{\left[-\frac{\hbar}{2 M} \nabla^{2}+\frac{1}{2} k\left(x^{2}+y^{2}\right)\right]+f\left(x \sigma_{z}-y \sigma_{x}\right)+g\left[\left(x^{2}-y^{2}\right) \sigma_{z}+2 x y \sigma_{x}\right]\right\}\left|\Psi_{i}(x, y)\right\rangle=\epsilon_{i}\left|\Psi_{i}(x, y)\right\rangle,
$$

where

$$
\sigma_{x}=|X\rangle\langle Y|+| Y\rangle\left\langle X\left|, \quad \sigma_{z}=\right| X\right\rangle\langle X|-| Y\rangle\langle Y|,
$$

and $f$ and $g$ are the linear and quadratic $\mathrm{JT}$ interaction parameters, respectively [1]. 
The two lowest-energy solutions of the JT equation are degenerate and have well-defined $E$ symmetry. Because our analysis is mostly performed in the low-temperature limit, we restrict ourselves to those two solutions and denote them

$$
\begin{aligned}
& \left|\Psi_{1}\right\rangle=|X\rangle \otimes\left|\chi_{1, x}\right\rangle+|Y\rangle \otimes\left|\chi_{1, y}\right\rangle, \\
& \left|\Psi_{2}\right\rangle=|X\rangle \otimes\left|\chi_{2, x}\right\rangle+|Y\rangle \otimes\left|\chi_{2, y}\right\rangle .
\end{aligned}
$$

We note that, although the vibronic states are normalized to 1 , the vibrational functions $\left|\chi_{1, x}\right\rangle$, etc., are not.

\section{CALCULATION OF HAM REDUCTION FACTOR}

We now use these vibronic solutions to construct the fine-structure states of the ${ }^{3} E$ manifold, which we use to calculate the Ham reduction factor. For example, the electronic state

$$
\left|A_{1}\right\rangle=\frac{1}{\sqrt{2}}\left(|X\rangle \otimes\left|S_{x}\right\rangle+|Y\rangle \otimes\left|S_{y}\right\rangle\right),
$$

which is an eigenstate of the NV center's intrinsic electronic Hamiltonian at low crystal strain and magnetic field, becomes

$$
\left|A_{1}^{\mathrm{JT}}\right\rangle=\frac{1}{\sqrt{2}}\left(\left|\Psi_{1}\right\rangle \otimes\left|S_{x}\right\rangle+\left|\Psi_{2}\right\rangle \otimes\left|S_{y}\right\rangle\right)
$$

when the JT interaction is taken into account, where $\left|S_{x}\right\rangle$ and $\left|S_{y}\right\rangle$ are the symmetrized $S=1$ spin states. The other five eigenstates of the ${ }^{3} E$ manifold can be constructed in a similar fashion. We note that, because the vibronic states $\left|\Psi_{1}\right\rangle$ and $\left|\Psi_{2}\right\rangle$ are degenerate in the absence of crystal strain and have the same $E$ symmetry as the orbital states $|X\rangle$ and $|Y\rangle$, this change preserves the familiar ${ }^{3} E$ fine structure and optical selection rules.

We now consider the axial spin-orbit interaction

$$
V_{\mathrm{SO}}^{\|}=L_{z} S_{z},
$$

where $L_{z}$ and $S_{z}$ are the axial orbital and spin angular momentum operators. We explicitly evaluate the expectation value of $V_{\mathrm{SO}}^{\|}$ corresponding to $\left|A_{1}^{\mathrm{JT}}\right\rangle$ to find the reduced axial spin-orbit coupling rate

$$
\lambda_{\|}^{\mathrm{JT}} \equiv\left\langle A_{1}^{\mathrm{JT}}\left|V_{\mathrm{SO}}^{\|}\right| A_{1}^{\mathrm{JT}}\right\rangle=p \lambda_{\|} .
$$

Here, we have introduced the unreduced axial spin-orbit coupling rate

$$
\begin{aligned}
\lambda_{\|} & =\left\langle A_{1}\left|V_{\mathrm{SO}}^{\|}\right| A_{1}\right\rangle=\left\langle A_{2}\left|V_{\mathrm{SO}}^{\|}\right| A_{2}\right\rangle \\
& =-\left\langle E_{1}\left|V_{\mathrm{SO}}^{\|}\right| E_{1}\right\rangle=-\left\langle E_{2}\left|V_{\mathrm{SO}}^{\|}\right| E_{2}\right\rangle
\end{aligned}
$$

and the Ham reduction factor

$$
p=\operatorname{Re}\left(\left\langle\chi_{1, x} \mid \chi_{2, y}\right\rangle-\left\langle\chi_{1, y} \mid \chi_{2, x}\right\rangle\right) \leqslant 1,
$$

which depends on the overlaps of the vibrational functions of $\left|\Psi_{1}\right\rangle$ and $\left|\Psi_{2}\right\rangle$. We see that in the absence of the JT interaction, $\left|\Psi_{1}\right\rangle$ and $\left|\Psi_{2}\right\rangle$ are the separable products of orbital and vibronic states given by Eq. (E3), and so

$$
\begin{aligned}
& \left\langle\chi_{1, x} \mid \chi_{2, y}\right\rangle=\left\langle\chi_{0} \mid \chi_{0}\right\rangle=1, \\
& \left\langle\chi_{1, y} \mid \chi_{2, x}\right\rangle=0,
\end{aligned}
$$

where $\left|\chi_{0}\right\rangle$ is the ground vibrational level calculated in the absence of the JT interaction. This leads to the expected result $p=1$.

We evaluate $p$ by using the values $f=-0.74 \mathrm{eV} / \AA, g=1.76 \mathrm{eV} / \AA^{2}$, and $k=14.5 \mathrm{eV} / \AA^{2}$ found by Abtew et al. [1] to solve Eq. (E4) numerically. We find the six lowest-energy solutions over a square region of side length $L=1 \AA$ using Dirichlet boundary conditions, which require that the vibrational wave function go to zero at the boundaries of the region. The calculated eigenenergies

$$
\epsilon_{i}=\{36.6,36.6,71.5,103.4,114.5,114.5\} \mathrm{meV}
$$

agree to better than $1 \%$ with those found by Abtew et al. The two lowest-energy solutions explicitly give $\left|\Psi_{1}\right\rangle$ and $\left|\Psi_{2}\right\rangle$, from which we can extract $p=0.43$.

The transverse spin-orbit coupling rate $\lambda_{\perp}$ that mediates the ISC transition from the spin-triplet $\left|A_{1}\right\rangle$ state to the spin-singlet $\left|{ }^{1} A_{1}\right\rangle$ state is also reduced by an analogous Ham reduction factor $q_{v_{n}}$, which can be calculated using a similar method. This reduction factor is given by

$$
q_{v_{n}}=\frac{\left\langle\chi_{v_{n}}^{\prime} \mid \chi_{1, x}\right\rangle+\left\langle\chi_{v_{n}}^{\prime} \mid \chi_{2, y}\right\rangle}{2\left\langle\chi_{v_{n}}^{\prime} \mid \chi_{0}\right\rangle}
$$

where $\left|\chi_{v_{n}}^{\prime}\right\rangle$ is a specific excited vibrational level of $\left|{ }^{1} A_{1}\right\rangle$ [cf. Eqs. (4) and (5)]. 


\section{IMPLICATIONS FOR ISC MODEL}

We now apply the Ham reduction factors $p$ and $q_{v_{n}}$ to our ISC rate calculation. First, the axial spin-orbit coupling rate that has been extracted from spectroscopy of the ${ }^{3} E$ manifold [4] is actually the reduced coupling rate $\lambda_{\| \mathrm{JT}}^{\mathrm{JT}}$. Our theoretical justification for $\lambda_{\|} \sim \lambda_{\perp}$ still holds and our bounds of $\lambda_{\perp} / \lambda_{\|}=1.2 \pm 0.2$ are still reasonable, but this comparison applies to the unreduced coupling rates. We therefore make the substitution $\lambda_{\perp} \rightarrow p^{-1} \lambda_{\perp}$ to account for the fact that we have used the experimentally measured $\lambda_{\|}^{\mathrm{JT}}$ as an input to our model.

We note that the fact that we have calculated a Ham reduction factor $p$ that is significantly less than 1 implies that the $a b$ initio calculations [5,6] upon which we have based the bounds of $\lambda_{\perp} / \lambda_{\|}$have likely underestimated the unreduced spin-orbit coupling rates. We assume that this underestimation applies equally to both $\lambda_{\perp}$ and $\lambda_{\|}$, but using further ab initio calculations to refine the ratio of these two parameters is currently an active area of research.

We also include Ham reduction factors $q_{v_{n}}$ by making the substitution $\lambda_{\perp} \rightarrow q_{v_{n}} \lambda_{\perp}$. Because the factor $q_{v_{n}}$ corresponds to a specific vibrational level of $\left|{ }^{1} A_{1}\right\rangle$, we may integrate it into the definition of the vibrational overlap function

$$
\begin{aligned}
F(\Delta) & =\sum_{n}\left|\left\langle\chi_{0} \mid \chi_{v_{n}}^{\prime}\right\rangle\right|^{2} q_{v_{n}}^{2} \delta\left(v_{n}-\Delta\right) \\
& =\overline{\left|\left\langle\chi_{0} \mid \chi_{\Delta}^{\prime}\right\rangle\right|^{2} q_{\Delta}^{2}} \rho(\Delta),
\end{aligned}
$$

which was previously given by Eq. (5). However, this reduction factor modifies the optical transition from the ${ }^{3} E$ manifold to the spin-triplet ${ }^{3} A_{2}$ ground state in the same way that it modifies the ISC to the spin-singlet $\left|{ }^{1} A_{1}\right\rangle$ state. Therefore, under our assumption that the ${ }^{3} A_{2}$ and $\left|{ }^{1} A_{1}\right\rangle$ states have similar vibrational spectra, the factor of $q_{v_{n}}^{2}$ that enters into our redefined $F(\Delta)$ is already included in the optical emission spectrum [7] that we use as another experimental input to our model. We therefore do not need to calculate and include the spectrum of $q_{v_{n}}$ explicitly.

Taken together, we must reduce the value of $F(\Delta)$ that enters into our expression for $\Gamma_{A_{1}}$ by $2 \pi$ to account for the normalization error, but we must multiply the factor of $\lambda_{\perp}^{2}$ by $p^{-2}$ in order to compensate for using the experimentally measured $\lambda_{\|}^{\mathrm{JT}}$. We therefore insert an overall correction factor

$$
\beta=\frac{p^{-2}}{2 \pi} \approx 0.86
$$

into our expression for $\Gamma_{A_{1}}$. Note that both errors enter into $\Gamma_{E_{1,2}}$ in the same way that they enter into $\Gamma_{A_{1}}$, so we need not correct our calculation of the ratio $\Gamma_{E_{1,2}} / \Gamma_{A_{1}}$.

The main change is to Fig. 5, which we reproduce as Fig. E5 with the original caption. Reducing the calculated value of $\Gamma_{A_{1}}$ by a factor of $\beta$ slightly shifts the regions to which we confine $\Delta$. The lower-energy intersection shifts upward from 43 to 46 meV and a new region appears around $87 \mathrm{meV}$. Both of these regions, however, are still excluded by our analysis of the $\Gamma_{E_{1,2}} / \Gamma_{A_{1}}$ ratio, which is still correct and still excludes points of intersection below $148 \mathrm{meV}$, as shown in Fig. 6(a). The higher-energy region of intersection now extends from $\Delta_{-}=321$ (344) $\mathrm{meV}$ to $\Delta_{+}=414$ (430) $\mathrm{meV}$ with a central value of $\Delta_{0}=376(392) \mathrm{meV}$, where the uncorrected values are given in parentheses.

There are other, even smaller, effects of this correction. Because our fitted range of $\Delta$ has changed slightly, the calculated ratio $\Gamma_{E_{1,2}} / \Gamma_{A_{1}}$, which is shown in Fig. 6(b) as a function of the acoustic phonon cutoff frequency $\Omega$, also changes slightly. We do not reproduce the figure here because the shift is small, but we note that our analysis now predicts that $\Omega$ is between 74 and

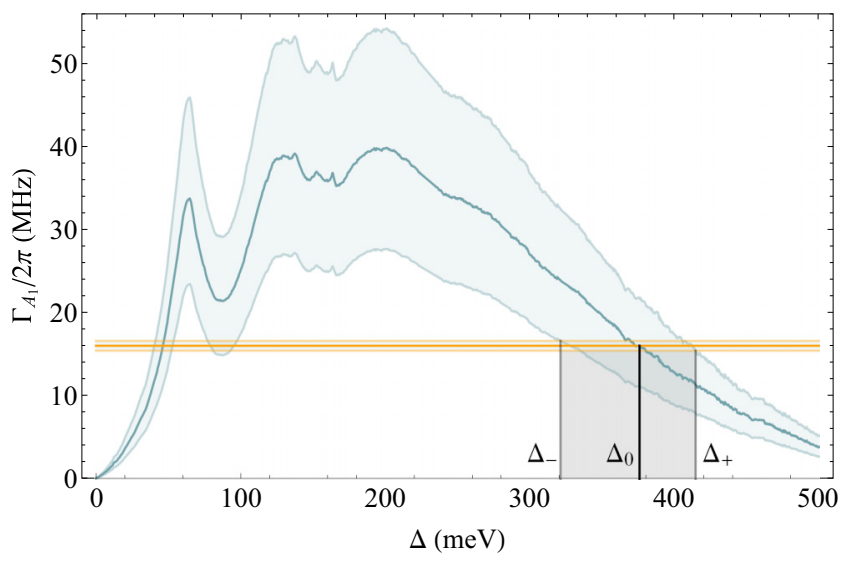

FIG. E5. ISC rate from $\left|A_{1}\right\rangle$. The values of $\Gamma_{A_{1}}$ calculated using Eq. (6) (blue) and measured in Ref. [8] (orange) are shown, with confidence intervals given by the uncertainty bounds on $\lambda_{\perp}$ described in the text and the $95 \%$ confidence interval given for the measurement. The predicted range of $\Delta$, which is defined by the intersection of the two curves, is shown in black. The points of intersection below 148 meV are excluded by the measured $\Gamma_{E_{1,2}} / \Gamma_{A_{1}}$ ratio, as explained in Sec. III B. 
$96 \mathrm{meV}$ rather than 74 and $93 \mathrm{meV}$. The plots in Fig. 7(b) shift by a similarly small amount, but the error in $\Gamma_{E_{1,2}} / \Gamma_{A_{1}}$ evaluated at the lower bound of the range of $\Omega$ and $T=25 \mathrm{~K}$ is still approximately $0.4 \%$. Similarly, the lifetime of the $m_{s}= \pm 1$ states that we project up to room temperature in Fig. 9 rises slightly as a result of the reduction of both $\Gamma_{A_{1}}$ and $\Gamma_{E_{1,2}}$ but it remains consistent with the measured lifetimes.

Overall, the essential results of the work are unchanged. Further, our inclusion of the Ham reduction factor into our model of the ISC mechanism reinforces the work's basic message, which is that interactions between lattice vibrations and the NV center's electronic orbital states impact the crucial ISC process in ways that are subtler than has been previously appreciated.

[1] T. A. Abtew, Y. Y. Sun, B.-C. Shih, P. Dev, S. B. Zhang, and P. Zhang, Dynamic Jahn-Teller Effect in the $\mathrm{NV}^{-}$Center in Diamond, Phys. Rev. Lett. 107, 146403 (2011).

[2] F. S. Ham, Effect of linear Jahn-Teller coupling on paramagnetic resonance in a ${ }^{2} E$ state, Phys. Rev. 166, 307 (1968).

[3] K.-M. C. Fu, C. Santori, P. E. Barclay, L. J. Rogers, N. B. Manson, and R. G. Beausoleil, Observation of the Dynamic Jahn-Teller Effect in the Excited States of NitrogenVacancy Centers in Diamond, Phys. Rev. Lett. 103, 256404 (2009).

[4] L. C. Bassett, F. J. Heremans, D. J. Christle, C. G. Yale, G. Burkard, B. B. Buckley, and D. D. Awschalom, Ultrafast optical control of orbital and spin dynamics in a solid-state defect, Science 345, 1333 (2014).
[5] M. W. Doherty, The theory of the nitrogen-vacancy colour centre in diamond, Ph.D. thesis, University of Melbourne, 2012.

[6] J. R. Maze, A. Gali, E. Togan, Y. Chu, A. Trifonov, E. Kaxiras, and M. D. Lukin, Properties of nitrogen-vacancy centers in diamond: the group theoretic approach, New J. Phys. 13, 025025 (2011).

[7] P. Kehayias, M. W. Doherty, D. English, R. Fischer, A. Jarmola, K. Jensen, N. Leefer, P. Hemmer, N. B. Manson, and D. Budker, Infrared absorption band and vibronic structure of the nitrogenvacancy center in diamond, Phys. Rev. B 88, 165202 (2013).

[8] M. L. Goldman, A. Sipahigil, M. W. Doherty, N. Y. Yao, S. D. Bennett, M. Markham, D. J. Twitchen, N. B. Manson, A. Kubanek, and M. D. Lukin, Phonon-Induced Population Dynamics and Intersystem Crossing in Nitrogen-Vacancy Centers, Phys. Rev. Lett. 114, 145502 (2015). 